\title{
Short communication: Influence of pulsed light treatment on the quality and sensory characteristics of Cheddar cheese
}

\author{
J. Proulx, M. Agustin, G. Sullivan, S. VanWees, J. Jian, S. T. Hilton, and C. I. Moraru' \\ Department of Food Science, Cornell University, Ithaca, NY 14853
}

\section{ABSTRACT}

The objective of this study was to examine the effect of pulsed light (PL) treatment on the color, oxidative stability, and onset of molding of Cheddar cheese. Slices of sharp white Cheddar cheese of $2.5 \times 5 \mathrm{~cm}$ were treated on one side with PL doses from 1.02 to $12.29 \mathrm{~J} /$ $\mathrm{cm}^{2}$, sealed in polyethylene bags, and stored at $6^{\circ} \mathrm{C}$ for up to 1 mo. Peroxide value, color parameters, and the onset of molding were evaluated. No significant changes in color or peroxide value were observed for PL-treated samples compared with the untreated controls. Pulsed light was able to significantly delay surface molding during refrigerated storage, with a PL dose of $9.22 \mathrm{~J} /$ $\mathrm{cm}^{2}$ delaying the onset of molding by $7 \mathrm{~d}$. The effect of PL on the taste, appearance, and acceptability of Cheddar cheese slices treated with a PL dose of $9.22 \mathrm{~J} /$ $\mathrm{cm}^{2}$ on each side was assessed. In triangle tests, $60 \mathrm{un}$ trained panelists were unable to detect significant differences between the control and PL-treated samples, although PL had a significant effect on overall liking, flavor, and appearance. These findings suggest that although PL can be effective for surface decontamination of cheese, it may have some detrimental effects on sensory properties.

Key words: pulsed light, cheese quality, sensory property, surface molding

\section{Short Communication}

Cheese products are susceptible to postprocessing contamination, which can lead to both food safety issues and significant losses due to spoilage (Nogarol et al., 2013; Gould et al., 2014). Out of the 90 cheese outbreaks reported in the United States from 1998 to $2011,49 \%$ were caused by cheese made from pasteurized milk (Gould et al., 2014) and several were caused by postpasteurization cross-contamination of cheese during cutting, slicing, or packaging, either at

Received June 7, 2016.

Accepted November 4, 2016.

${ }^{1}$ Corresponding author: cim24@cornell.edu the processing plant or in retail environments. Many of these outbreaks involved the gram-positive Listeria monocytogenes and the gram-negative Escherichia coli O157:H7 (Gould et al., 2014). The main approach to prevent postpasteurization microbial contamination of cheese is maintaining good manufacturing practices and proper sanitation. Many cheese are also vacuum-packed to prevent the growth of contaminating microflora during refrigerated storage. Besides these measures, an additional microbial inactivation step that targets the contaminating surface microflora after processing, handling, and packaging of cheese could be extremely beneficial.

Pulsed light (PL) treatment, which consists of short, high-energy pulses of broad-spectrum light containing wavelengths ranging from UV to near-infrared, was explored recently as a possible solution to address this issue (Proulx et al., 2015). Pulsed light is approved by the US Food and Drug Administration for the decontamination of food and food contact surfaces at doses not exceeding $12 \mathrm{~J} / \mathrm{cm}^{2}$ (FDA, 1996). Pulsed light was shown to effectively inactivate pathogenic and spoilage microorganisms on the cheese surface, with $1.41 \pm 0.15$ $\log$ reductions of $L$. monocytogenes on American cheese (Can et al., 2014), almost 3 log reductions of Listeria innocua, more than $5 \log$ reduction of Escherichia coli, and more than $3.5 \mathrm{log}$ reduction of Pseudomonas fluorescens on Cheddar cheese (Proulx et al., 2015) being reported. Pulsed light has also been shown to be effective against a variety of yeasts and molds (Elmnasser et al., 2007; Gomez-Lopez et al., 2007). Proulx et al. (2015) also evaluated the effect of bacterial load, cheese surface topography, and the presence of clear polyethylene packaging on the effectiveness of PL for cheese surface decontamination.

Although PL is an attractive solution from a microbiological inactivation perspective, limited data are available on its influence on the quality and organoleptic properties of cheese. Due to its spectral makeup, it is possible that PL would affect cheese quality. For instance, light is known to be detrimental to the quality of milk and other dairy products by initiating lipid oxidation, which can subsequently lead to product 
discoloration and the development of rancid off-flavors during storage (Kristensen et al., 2000). Light is also notorious for its ability to degrade light-sensitive micronutrients, such as riboflavin (Deger and Ashoor, 1987). Therefore, the objective of our study was to assess the effect of PL on color change, oxidative stability, and onset of molding on Cheddar cheese, and, through sensory tests, to determine whether PL affects the appearance, taste, and acceptability of Cheddar cheese slices. Additionally, the ability of PL to extend shelf life of cheese by preventing or delaying surface molding was also evaluated.

Pulsed light treatments were conducted using a Xenon RS-3000C unit (Xenon Corporation, Wilmington, MA) equipped with a Xenon flash lamp emitting 360$\mu$ s pulses at 3 pulses per second. The PL dose (fluence) measurements were taken using a pyroelectric head (PE25BB-DIF) coupled with a Nova II display (Ophir Optronics Inc., Wilmington, MA). During the measurement, the pyroelectric head and its chord were covered in aluminum foil, leaving only the diffuser opening exposed. Cumulative exposure was measured at the same distance from the lamp housing $(5.8 \mathrm{~cm})$ as the samples, at a pulse width setting of $1.0 \mathrm{~ms}$. All fluence measurements were taken in triplicate. At least 1 min was allowed between measurements to prevent overheating of the pyroelectric head. The experimental conditions were chosen based on previous work on surface decontamination of cheese by PL treatment (Proulx et al., 2015).

Eight-ounce (227 g) blocks of vacuum-packaged sharp white Cheddar cheese (Heluva Good, Sodus, NY) were purchased from a local retail store (Tops, Ithaca, NY) and kept under refrigeration conditions until use. Rectangular cheese slices of $2.5 \times 5 \mathrm{~cm}$ (width $\times$ length) and 1 to $3 \mathrm{~mm}$ thick were obtained from the 8-ounce $(227 \mathrm{~g})$ cheese blocks using a sterilized cheese cutter. The exact weight of each slice was recorded and samples of $4 \pm 0.2 \mathrm{~g}$ were used for the experiments. For each treatment, an individual cheese sample was placed at $5.8 \mathrm{~cm}$ below the surface of the lamp housing. Cheese samples were subjected to $1,3,6,9$, or 12 pulses, corresponding to fluence levels of $1.02,3.07,6.14,9.22$, and $12.29 \mathrm{~J} / \mathrm{cm}^{2}$, respectively. For all tests, cheese samples that were not treated with PL were used as controls.

For peroxide value monitoring and sensory evaluation, cheese slices were subjected to a PL dose of 9.22 $\mathrm{J} / \mathrm{cm}^{2}$, which is representative of the plateau in the microbial inactivation curve (Proulx et al., 2015). After PL treatment, each cheese slice was sealed in an individual polyethylene bag (Ziploc, SC Johnson, Racine, WI) and stored inside a commercial dairy display case equipped with a front low-UV emitting T-8 fluorescent lamp (True Manufacturing, O'Fallon, MO). The storage temperature was set at $6 \pm 1^{\circ} \mathrm{C}$ and the samples were kept for up to $30 \mathrm{~d}$.

Lipid peroxides were used as indicators for early-stage oxidative changes (Shahidi and Zhong, 2005). In our study, peroxide value (PV) was monitored spectrophotometrically using the International Dairy Federation Standard method 74A (IDF, 1991). In this method, lipid peroxides oxidize ferrous ions to ferric ions, which then form a ferric-thiocyanate complex that absorbs at $500 \mathrm{~nm}$. The PV development was measured 3 times a week over $30 \mathrm{~d}$ of refrigerated storage on untreated Cheddar and PL-treated cheese subjected to $9.22 \mathrm{~J} /$ $\mathrm{cm}^{2}$. The lipid portion of cheese was extracted using a method adapted from Kristensen et al. (2001). Cheese samples were first individually grated and transferred to a beaker containing $80 \mathrm{~mL}$ of chloroform-methanol $(2: 1 \mathrm{vol} / \mathrm{vol})$. The mixture was homogenized using an IKA Ultra-Turrax homogenizer (IKA, Wilmington, DE) at 7,000 rpm for $60 \mathrm{~s}$. After $\mathrm{CaCl}_{2}$ addition (1.0 $\mathrm{m} M ; 16 \mathrm{~mL}$ ) and a vigorous 15 -s shake, the mixture was centrifuged at $500 \times g$ for 30 min using a Sorvall RC5B Refrigerated Superspeed Centrifuge (Thermo Scientific, Waltham, MA). The sediment (bottom layer) was transferred to an evaporation flask and left to dry under a vacuum at room temperature until all the chloroform evaporated. Measurements were performed in triplicate on samples obtained from 3 separate PL treatments. Peroxide values were reported as milliequivalents of $\mathrm{O}_{2}$ per gram of lipid.

The color of each cheese slice was measured before PL treatment, immediately after PL treatment, and then every 2 d over a 30-d refrigerated storage period. A Chroma C-400 colorimeter (Konica Minolta, Chiyoda, Japan) was used after proper calibration. Color measurements were taken through the transparent polyethylene bag in which samples were stored to minimize sample manipulations. Color change was expressed as CIELAB $L^{*}, a^{*}, b^{*}$ values, where $L^{*}$ is a measure of increasing lightness ranging from 0 to $100, a^{*}$ is a measure of the sample's color on the Comission Internationale de $l^{\prime}$ Eclairage (CIE) green-red axis, and $b^{*}$ is a measure of the sample's position on the CIELAB blue-yellow axis (Witzel et al., 1973). The distance metric $\Delta \mathrm{E}$ was also calculated as a more global measure of perceptual color change, according to the formula (Lawless and Heymann, 2010):

$$
\Delta \mathrm{E}=\sqrt{(\Delta \mathrm{L} *)^{2}+(\Delta \mathrm{a} *)^{2}+(\Delta \mathrm{b} *)^{2}} .
$$

All measurements were performed in triplicate on samples obtained from 3 separate PL treatments. 
To evaluate the onset of molding, cheese slices were cut from the outer portion of the commercial bricks of Cheddar cheese to ensure that the surface exposed to PL treatment had been previously exposed to typical handling conditions in a commercial cheese making facility. Recorded mold development thus originated from spores present in the processing environment and subsequent handling. A set of 30 cheese slices was prepared for each PL treatment dose. The onset of molding was monitored daily by visually inspecting the samples; the time until the first appearance of mold growth on each cheese slice was recorded. The time until onset of molding on PL-treated Cheddar cheese was estimated using survival analysis, a biostatistics method identified as a useful tool for food shelf life studies (Marquenie et al., 2002). This method is able to account for incomplete data sets (Kleinbaum and Klein, 2005), which was necessary in our study given that not all cheese slices had molded by the time the study ended. The nonparametric Kaplan-Meier estimator, $\hat{\mathrm{S}}(\mathrm{t})$, which approximates the probability of a cheese sample to remain mold-free until a certain time, t, was calculated and used to describe general molding patterns of PL-treated cheese. A semiparametric Cox proportional hazards model was then used to assess the effect of PL dose on the onset of molding. A goodness of fit test based on Schoenfelds' residuals confirmed that the Cox proportional hazards assumptions were respected. Survival analysis was performed using Small Stata 13.1 (StataCorp, College Station, TX).

Sensory tests were performed using a panel of 60 untrained panelists. Samples for the sensory evaluation were treated with $9.22 \mathrm{~J} / \mathrm{cm}^{2}$ on both sides of the slice to ensure that the panelists always placed a treated side on their tongue. Two days before sensory testing, the 8 -ounce $(227 \mathrm{~g})$ cheese blocks were cut into $2.5 \times$ $5 \mathrm{~cm}$ slices of $0.5 \mathrm{~cm}$ thickness and treated with PL. Treated samples and untreated controls were placed in cups labeled with random 3-digit identification codes and arranged on trays. The trays were wrapped with plastic wrap, to prevent drying of the samples, and stored at $4^{\circ} \mathrm{C}$ until next day. Samples were taken out of the refrigerator $1 \mathrm{~h}$ before serving and allowed to equilibrate to room temperature. All panelists received samples at approximately the same temperature. Each panelist completed a triangle test and a liking test comparing PL-treated to control samples. During the triangle test, panelists were presented with a group of samples composed of 2 samples of the same treatment and 1 different sample. The panelists were then asked to indicate which of the 3 samples was different. During the liking test, panelists were asked to rate the products on a 9-point hedonic scale. Each sample was assigned a random 3-digit code and the order in which the samples were presented was randomized. Panelists completed their tests on a computer ballot in individual booths under white lighting. Data were collected and analyzed using Compusense 5 version 4.6 (Compusense Inc., Guelph, ON, Canada).

Statistical analyses was conducted for all data. A oneway ANOVA was performed on $\triangle \mathrm{E}$-CIELAB values for each day of storage. If a significant effect of PL dose on $\Delta \mathrm{E}$ was found significant on a given day, Tukey's honest significant difference (HSD) test was carried out to determine which factor level differed from the others. The same procedure was followed to determine significant differences in peroxide values and in overall liking, flavor, and appearance between untreated and PL-treated Cheddar samples. Data were analyzed using the statistical package JMP Pro 10 (SAS Institute Inc., Cary, NC).

The lightness parameter $\mathrm{L}^{*}$ for PL-treated samples remained stable over a 30-d refrigerated storage period, at approximately 75 . The green-red hue increased slightly, moving toward neutrality, and the yellow-blue hue decreased slightly. It is important to note that these slight changes in hue did not translate into a perceptible change in color to the naked eye. This is consistent with other work, where authors were only able to see significantly altered cheese color at an elevated storage temperature of $37^{\circ} \mathrm{C}$ (Kristensen et al., 2001). No significant differences $(P<0.05)$ in $\Delta \mathrm{E}$ were observed between PL-treated cheese samples and untreated controls. The only exception was a significant difference found after $10 \mathrm{~d}$ of refrigerated storage, where samples treated with $12.29 \mathrm{~J} / \mathrm{cm}^{2}$ had $\Delta \mathrm{E}$ values significantly different from the untreated controls (Table 1). This single discrepancy did not reflect the overall trend though.

The untreated controls experienced a PV increase from $0.07 \pm 0.03$ at $\mathrm{d} 0$ to $0.19 \pm 0.05$ after a month of refrigerated storage in a commercial dairy case illuminated with fluorescent light. No significant increase

Table 1. The distance metric $\Delta \mathrm{E}$ values (a measure of color change) of pulsed light (PL)-treated Cheddar cheese after $10 \mathrm{~d}$ of refrigerated storage

\begin{tabular}{lc}
\hline PL dose $\left(\mathrm{J} / \mathrm{cm}^{2}\right)$ & $\Delta \mathrm{E}$ \\
\hline 0 (control) & $1.70 \pm 0.40^{\mathrm{a}}$ \\
1.02 & $3.38 \pm 0.67^{\mathrm{b}}$ \\
3.07 & $2.63 \pm 0.31^{\mathrm{ab}}$ \\
6.14 & $2.21 \pm 0.72^{\mathrm{ab}}$ \\
9.22 & $3.13 \pm 0.77^{\mathrm{ab}}$ \\
12.29 & $1.79 \pm 0.41^{\mathrm{b}}$
\end{tabular}

${ }^{\mathrm{a}, \mathrm{b}}$ Values marked with different letters are significantly different from each other $(P<0.05)$. 
Table 2. Mean hedonic scores $( \pm 1 \mathrm{SD})$ on a 9-point scale ${ }^{1}$ for Cheddar cheese samples treated with pulsed light (PL), at a dose of $9.22 \mathrm{~J} / \mathrm{cm}^{2}$ per side, versus untreated controls

\begin{tabular}{lcc}
\hline Sensory attribute & $\begin{array}{c}\text { Untreated } \\
\text { control }\end{array}$ & $\begin{array}{c}\text { PL } \\
\text { treated }\end{array}$ \\
\hline Overall liking & $6.6 \pm 1.3^{\mathrm{a}}$ & $4.9 \pm 1.9^{\mathrm{b}}$ \\
Overall flavor & $6.5 \pm 1.4^{\mathrm{a}}$ & $4.5 \pm 2.0^{\mathrm{b}}$ \\
Overall appearance & $6.8 \pm 1.3^{\mathrm{a}}$ & $5.8 \pm 1.6^{\mathrm{b}}$ \\
\hline
\end{tabular}

${ }^{\mathrm{a}, \mathrm{b}}$ Values marked with different letters are significantly different from each other $(P<0.05)$.

${ }^{1}$ Nine-point hedonic scale: $1=$ dislike extremely, $2=$ dislike very much, $3=$ dislike moderately, $4=$ dislike slightly, $5=$ neither like or dislike, $6=$ like slightly, $7=$ like moderately, $8=$ like very much, $9=$ like extremely.

in $\mathrm{PV}(P<0.05)$ compared with controls was observed for PL-treated Cheddar slices. For samples treated with $9.22 \mathrm{~J} / \mathrm{cm}^{2}, \mathrm{PV}$ increased from $0.09 \pm 0.02$ to $0.23 \pm$ 0.02 after $30 \mathrm{~d}$ of storage. These results show no significant effect of PL dose on lipid peroxide formation in Cheddar cheese. By contrast, Kristensen et al. (2001) reported significantly higher PV for processed cheese samples exposed to fluorescent light at a 2,000 lx intensity after $1 \mathrm{mo}$ of storage at $5^{\circ} \mathrm{C}$ compared to untreated samples, with PV quickly exceeding 1 . It must be noted though that, as PL is a surface treatment, an important parameter that could change its effect on cheese oxidative stability is the surface-to-volume ratio of the samples, with higher surface area being more conducive of quantifiable oxidative effects.

Sensory analyses revealed some differences between PL-treated and untreated cheese samples. The hedonic scores given by the 60 panelists shows that PL-treated samples did have significantly lower scores on overall liking, flavor, and appearance as compared with the untreated controls (Table 2). Also, according to the distribution of the scores, panelists have consistently scored the PL-treated samples lower than the untreated samples. Interestingly though, in triangle tests that included the PL-treated samples and the untreated controls, only 26 out of 60 panelists $(43 \%)$ chose the correct sample, which means that at a $95 \%$ significance level panelists were unable to detect a difference between the untreated and PL-treated samples. Of the panelists that correctly identified the PL-treated sample from the 2 control samples, some noticed an aftertaste, others a cooked or oxidized flavor or, more generically, a difference in aroma. It should be noted that the PL treatment dose used here was rather aggressive, as both sides of the thin cheese slices were treated. Undesirable sensory effects could be minimized in commercial applications by applying PL treatment to the surface of large blocks of cheese rather than on very thin slices.

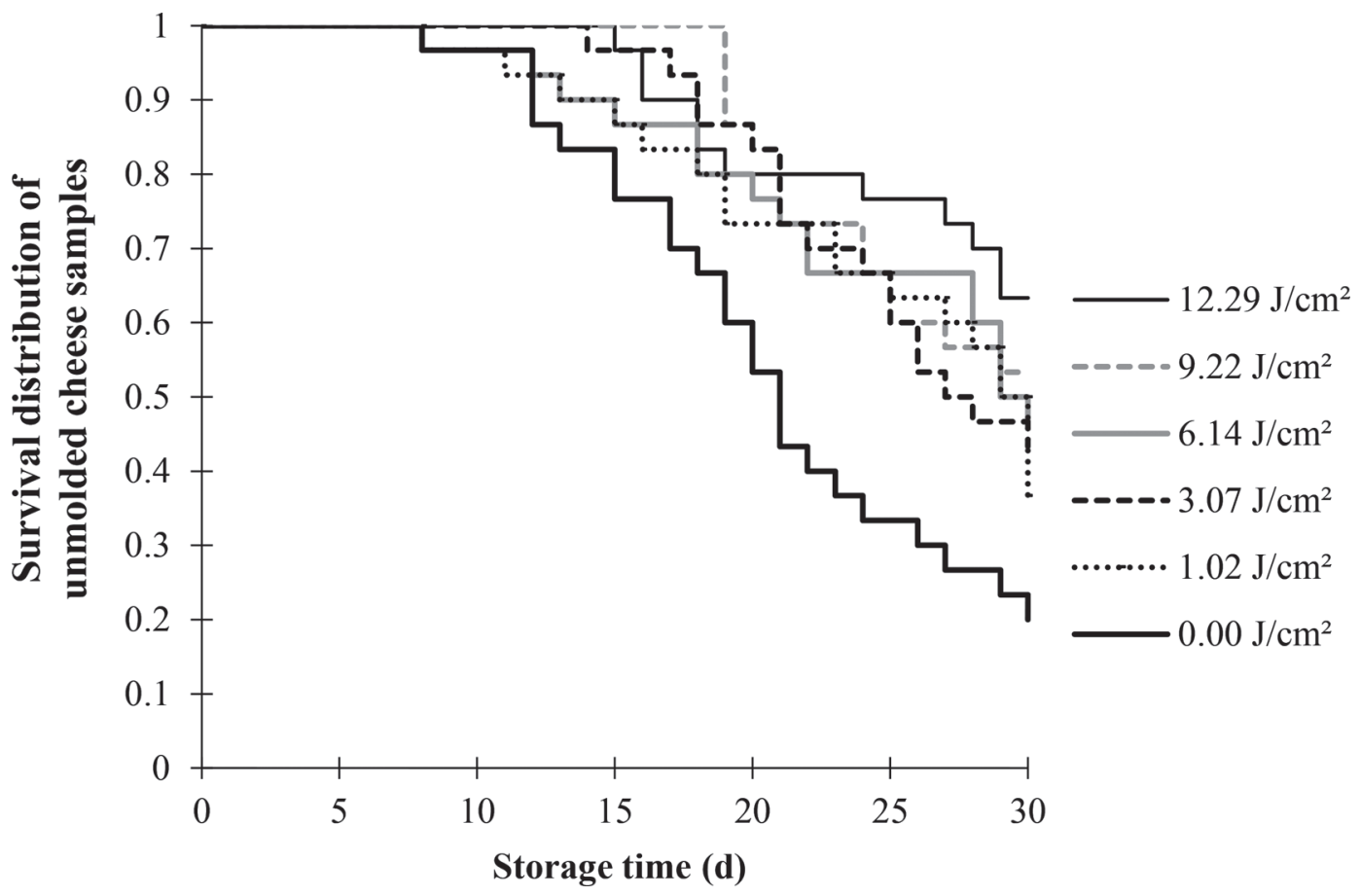

Figure 1. Kaplan-Meier survival curves of 180 Cheddar samples treated with varying pulsed light doses. Each curve associated with a particular dose represents the onset of molding in a set of 30 Cheddar slices treated under the same conditions. (Survival of $1=0 \%$ of cheese samples are molded, Survival of $0=100 \%$ of cheese samples are molded.) 
Survival analysis was used to evaluate the time until the first mold growth on PL-treated Cheddar cheese occurred revealed that a PL dose of $9.22 \mathrm{~J} / \mathrm{cm}^{2}$ could delay the onset of mold appearance by $7 \mathrm{~d}$ compared with the control (Figure 1). The median survival times obtained from the Kaplan-Meier estimates also reveal that after $21 \mathrm{~d}$ half of the control samples were moldy. In contrast, it took 28 or $30 \mathrm{~d}$ for samples treated at low to medium PL doses for half the samples to mold, whereas PL doses of 9.22 and $12.29 \mathrm{~J} / \mathrm{cm}^{2}$ caused more than half of the samples to remain mold-free after the 1-mo refrigerated storage period. A Cox proportional hazard model was used to establish whether these apparent differences between the controls and PL-treated Cheddar slices were significant. A univariate Wald test revealed that PL dose had a highly significant effect on the time until onset of molding $(P<0.001)$. With a hazard ratio of 0.93 , for every 1 unit increase in PL dose the risk of obtaining moldy cheese is reduced by $107 \%$. This could result in shelf life extension of cheese in the distribution chain, even at low PL doses. Nonetheless, a cost-benefit analysis would be required to assess the feasibility of adding PL as an additional hurdle to current processing lines.

Overall, our study showed that PL treatment, besides improving microbial safety of cheese, can have additional shelf life benefits. Specifically, a treatment dose in the plateau range of PL inactivation curves for several food-borne bacteria was also able to delay onset of surface molding of Cheddar cheese by $7 \mathrm{~d}$ and also slow down the rate of molding. Although no significant changes in color or lipid oxidation were observed for PL-treated samples during a month of refrigerated storage, a dose of $9.22 \mathrm{~J} / \mathrm{cm}^{2}$ had a negative effect on the organoleoptic properties of cheese. Therefore, PL treatment dose and mode of application should be selected strategically to maximize its antimicrobial benefits while minimizing its potential detrimental effects. For instance, the negative effects on the sensory attributes of cheese might be mitigated by surface application on large cheese blocks rather than on thin slices.

\section{ACKNOWLEDGMENTS}

This project was supported by the Dairy Research Institute (Project no. DRI-65385; Rosemont, IL).

\section{REFERENCES}

Can, F. O., A. Demirci, V. M. Puri, and H. Gourama. 2014. Decontamination of hard cheeses by pulsed UV light. J. Food Prot. 77:1723-1731.

Deger, D., and S. H. Ashoor. 1987. Light-induced changes in taste, appearance, odor, and riboflavin content of cheese. J. Dairy Sci. 70:1371-1376.

Elmnasser, N., S. Guillou, F. Leroi, N. Orange, A. Bakhrouf, and M. Federighi. 2007. Pulsed-light system as a novel food decontamination technology: A review. Can. J. Microbiol. 53:813-821.

FDA (US Food and Drug Administration). 1996. Kinetics of Microbial Inactivation for Alternative Food Processing Technologies--Pulsed Light Technology, final rule. 21 CFR part 179.41. Accessed Mar. 3 2013. http://www.ecfr.gov/cgi-bin/textidx?SID $=57$ af6cfd4fc 81035 640c1c1c78b95f19\&node=pt21.3.179\&rgn=div5\#se21.3.179_141.

Gomez-Lopez, V. M., P. Ragaert, J. Debevere, and F. Devileghere. 2007. Pulsed light for food decontamination: A review. Trends Food Sci. Technol. 18:464-473.

Gould, L. H., E. Mungai, and C. Barton Behravesh. 2014. Outbreaks attributed to cheese: Differences between outbreaks caused by unpasteurized and pasteurized dairy products, United States, 19982011. Foodborne Pathog. Dis. 11:545-551.

IDF. 1991. Anhydrous milkfat. Determination of peroxide value. Standard 74A. International Dairy Federation (IDF), Brussels, Belgium.

Kleinbaum, D. G., and M. Klein. 2005. Survival Analysis. 2nd ed. Springer, New York, NY.

Kristensen, D., E. Hansen, A. Arndal, R. A. Trinderup, and L. H. Skibsted. 2001. Influence of light and temperature on the colour and oxidative stability of processed cheese. Int. Dairy J. 11:837843.

Kristensen, D., V. Orlien, G. Mortensen, P. Brockhoff, and L. H. Skibsted. 2000. Light-induced oxidation in sliced havarti cheese packaged in modified atmosphere. Int. Dairy J. 10:95-103.

Lawless, H. T., and H. Heymann. 2010. Sensory Evaluation of Food 2nd ed. Springer, New York, NY.

Marquenie, D., C. W. Michiels, A. H. Geeraerd, A. Schenk, C. Soontjens, J. F. Van Impe, and B. M. Nicolaï. 2002. Using survival analysis to investigate the effect of UV-C and heat treatment on storage rot of strawberry and sweet cherry. Int. J. Food Microbiol. 73:187-196.

Nogarol, C., P. L. Acutis, D. M. Bianchi, C. Maurella, S. Peletto, S. Gallina, and L. Decastelli. 2013. Molecular characterization of Pseudomonas fluorescens isolates involved in the Italian "blue mozzarella" event. J. Food Prot. 76:500-504.

Proulx, J., L. C. Hsu, B. M. Miller, G. Sullivan, K. Paradis, and C. I. Moraru. 2015. Pulsed light inactivation of pathogenic and spoilage bacteria on cheese surface. J. Dairy Sci. 98:5890-5898.

Shahidi, F., and Y. Zhong. 2005. Lipid oxidation: Measurement methods. Pages 357-385 Bailey's Industrial Oil and Fat Products. John Wiley \& Sons Inc., Hoboken, NJ.

Witzel, R. F., R. W. Burnham, and J. W. Onley. 1973. Threshold and suprathreshold perceptual color differences. J. Opt. Soc. Am. 63:615-625. 